\title{
Die Bedeutung des Wortes
}

\author{
J. Horn
}

Abteilung für Allgemein- und Viszeralchirurgie, Städtisches Krankenhaus München-Harlaching, Deutschland

Die Z eit, in der wir leben, ist kurzatmig, schnelllebig, ungeduldig, von Augenblick zu Augenblick hastend. Wir betreiben die Dinge und die Dinge, die wir bewegen, treiben uns. Jede Frage sucht ihre schnelle A ntwort, jedes Problem seine entlastende Lösung. Wir haben verlernt, das A ufgehen des Samens geduldig zu erwarten, die innere Erregung zuzulassen, die der E ntfaltung der B lüte entgegensieht, und schließlich dem Wachsen die Zeit des R eifens zuzugestehen.

Stattdessen erleben wir eine $E$ intönigkeit der $\mathrm{G}$ efühle, die sich nur noch dem $R$ ausch und der schnellen B efriedigung hingeben; wir erleben eine Stumpfheit der Sinne, die sich an der L ust des Ä ußeren und dem Blendwerk des Scheins ergötzt; wir erleben eine $O$ rientierungslosigkeit, die im $\mathrm{D}$ enken und $\mathrm{H}$ andeln nur noch opportunistisch auf Vorteil bedacht ist; wir erleben eine Fülle des A ngebots, ein Ü bermaß an Möglichkeiten und wir merken nicht, wie sich der $M$ angel in unseren $\mathrm{H}$ erzen ausbreitet und dabei das Innere wie ein $\mathrm{K}$ rebsgeschwür zerstört.

In allen Bereichen unseres gesellschaftlichen Lebens ist dieser fressende und zerstörende Prozess spürbar und nur manchmal, aufgeschreckt durch besonders widrige und auffällige Vorkommnisse, fragen wir uns entrüstet, wie das denn sein könne, wie es möglich war, uns so weit von den Idealen und Werten menschlicher Tugenden zu entfernen.

Wir beklagen den Z ustand und tun doch alles, uns in seine Verhältnisse gefügig einzubringen. Wir beklagen den Mangel an Vorbildern und tun doch alles, um jeder Nivellierung Vorschub zu leisten. Wir beklagen den wirtschaftlichen Niedergang und kolportieren doch jeden Erfolg mit der neidvollen Vorstellung von der G leichheit des A nspruchs. Wir rätseln über den Niedergang von Kultur und Bildung und doch lassen wir es zu, dass dem hohen A nspruch der Bildung die Fesseln des kurzlebig Funktionalen angelegt werden. Wir beklagen den Verlust an L ebensfreude und Spontaneität, doch wir lassen es zu, dass jede I nitiative und jede $\mathrm{K}$ reativität den Kontrollmechanismen der Bürokratie geopfert wird.

Für jede Entwicklung, wie auch immer sie geartet sei, gibt es Indikatoren und M essgrößen, die R ichtung und A usmaß, Schwere und Verlust abschätzen lassen. E iner dieser Indikatoren ist die B edeutung des Wortes.
Nun scheint kein Z weifel darin zu bestehen, dass wir es mit einer inflationären Entwicklung des Wortes zu tun haben. Allein die Vielzahl der Worte, die uns täglich in R undfunk und Fernsehen, im Internet, in Z eitungen und J ournalen, in Werbespots und plakativen Parolen und darüber hinaus in platter Verfremdung und Verunstaltung zu G ehör kommen, zeigt neben der überflutenden Fülle die Lautstärke und die Dominanz dieser überhand nehmenden Nichtigkeiten. Während die Worte geradezu sintflutartig und in zunehmender Bedeutungslosigkeit über uns hereinbrechen, ist gleichzeitig ein wachsendes Bedürfnis festzustellen, sich in diesem R ausch der B elanglosigkeiten aufzuhalten, sich in ihm zu vergnügen und die eigene Person im M urmelspiel der Worthülsen zu spiegeln.

A uf der jährlich abgehaltenen B uchmesse erblicken bis zu 350000 Neuerscheinungen das Licht der Welt. Dabei muss offen bleiben, ob diese Inflation des Wortes A usdruck der Fülle oder vielleicht doch eher A usdruck eines $M$ angels ist. Wir müssen feststellen, dass gerade diejenigen A ttribute des Wortes Platz ergriffen haben, die in einem gewissen Widerspruch zu seiner Bedeutung stehen: $L$ autstärke, plakativer H ochglanz und Q uantität.

Wie weit haben wir uns von dem Wort entfernt, das am A nfang stand, das groß war und bedeutungsvoll genug, die Schöpfung entstehen zu lassen (J ohannes E vangelium 1,1). I m Wort lag die K raft, aus der alles entstanden ist, seine Bedeutung war es, die alles erschuf und seine Verlässlichkeit ist es, die alles erhält, bewahrt und trägt.

Bevor aber die Verfremdung und der M issbrauch des Wortes verstanden werden kann, muss versucht werden, der eigentlichen Bedeutung des Wortes näher zu kommen. Wie kann es denn sein, dass das Wort am A nfang der Schöpfung stand. Wohlgemerkt nicht Worte oder ein Wort, sondern das Wort! In der Tat geht es nicht um die Begrifflichkeit eines Wortes oder der Worte, vielmehr geht es um das Wort, welches gegeben wird, um G laubwürdigkeit zu bekunden, um Echtheit zu besiegeln und die Einzigartigkeit eines Bundes zu schließen.

E s fällt dem M enschen schwer, sich dieser tieferen Bedeutung des Wortes anzunähern, hält er es doch eher mit den Dingen, die ihm vorstellbar sind - so etwa, wenn Faust in seinem Studierzimmer die

$\begin{array}{ll}\text { KARGER } & \odot \text { 2004 S. K arger G mbH , Freiburg } \\ \text { Fax }+497614520714 & \text { A ccessible online at: } \\ \begin{array}{l}\text { E-mail Information@K arger.de } \\ \text { www.karger.com }\end{array} & \text { www.karger.com/journals/cga }\end{array}$

Prof. D r. Johannes H orn

A bteilung für A llgemein- und V iszeralchirurgie

Städtisches $\mathrm{K}$ rankenhaus $\mathrm{M}$ ünchen- $\mathrm{H}$ arlaching

Sanatoriumsplatz 2, D-81545 M ünchen

Tel. +498962 10-2301, Fax -2938

E-mail j.horn@khmh.de 
verschiedenen Möglichkeiten durchdenkt: «Im A nfang war das Wort!» U nd er sagt: «I ch kann das Wort so hoch unmöglich schätzen ...». «lm A nfang war der Sinn?» «Im A nfang war die K raft?» «Im A nfang war die Tat?»

Wie aber verhält es sich mit der K raft und der Tat, die G oethe als Möglichkeit des A nfangs ins A uge fasste? Beide, Kraft und Tat, beziehen sich ausschließlich auf die U msetzung von A bsicht und Willen, und sie enden, wenn das Werk abgeschlossen ist. I $m$ U nterschied zu dieser prometheischen Deutung des A nfangs beinhaltet das am A nfang stehende Wort weit mehr als K raft, Tat und Sinn, schon allein dadurch, dass sich mit dem Wort das A ngesprochenWerden und das A ngesprochen-Sein verbindet, wodurch ein persönlicher B ezug hergestellt wird.

E s wird deutlich, dass es um die A uthentizität geht, die dem Wort die herausragende B edeutung verleiht. D ie E inheit von U rsprung und Sein, von Wort und Tat, von A ussage, Versprechen und E rfüllung, von Gestaltung und Beständigkeit, die E inheit von Leben und Sein. D ieses Wort ist in die Zeit hineingesprochen, in eine sich ständig verändernde Welt, in eine Welt des Werdens und Vergehens. $M$ it seiner U nwiderlegbarkeit und seiner A -priori-G ültigkeit hebt es die Z eitlichkeit auf und stellt damit die Z usage des L ebens gegen die Vergänglichkeit und den Tod.

Weil es so ist, weil dieses gegebene Wort den A nfang der Schöpfung und den A nfang allen Seins markiert, ist es nicht veränderbar, nicht auflösbar, es ist nicht beliebig auslegbar. E s gibt keine Z weideutigkeit, keine Zufälligkeit, und es gibt keinen $E$ rsatz und keine A Iternative. D ies alles trägt dazu bei, dass das Wort als die M arkierung des A nfangs nicht umzudeuten und nicht wegzudenken ist. $A$ ber es ist nicht nur Markierung, vielmehr ist es in dieser $U$ rheberschaft und in seiner Letztgültigkeit die $\mathrm{K}$ raft, in der sich alles entfalten und gestalten kann, was ist; in der die E nergie des Schaffens, E ntstehens und des Werdens verborgen liegt.

$A$ ber es ist nicht nur $M$ arkierung und nicht nur die $K$ raft des Schaffens und des Gestaltens, die dieses Wort einzig und groß macht, vielmehr ist es der in ihm enthaltene Sinn, der das Schaffen mit Inhalten füllt, der die K räfte ordnet und dem Werden die R ichtung gibt. D och auch dies reicht nicht aus, die umfassende B edeutung dieses Wortes zu erhellen; nicht nur M arkierung des A nfangs, nicht nur schaffende K raft und nicht nur O rientierung und Sinn. V ielmehr ist es Versprechen und Verlässlichkeit, es ist die U nwiderruflichkeit einer Z usage, es ist gleichsam ein I dentitätsmerkmal für alles L ebendige, für alles, was Teil dieser Schöpfung ist.

D as Wort stellt eine Verbindung her zwischen dem, der es sagt und dem, der angesprochen ist. Die A uthentizität und die Verlässlichkeit des Wortes sind wesentliche Voraussetzungen für die G laubwürdigkeit dessen, der das Wort spricht. Nachdem nun der A ngesprochene Teil dieser Verbindung ist, die das Wort herstellt, wird es auch von ihm abhängen, inwieweit aus der Verbindung ein tragfähiges Verhältnis bzw. ein verlässlicher B und wird.

Verlassen wir die Einmaligkeit und E inzigartigkeit dieses am A nfang der Schöpfung stehenden Wortes und wenden uns den heute üblichen U mgangs- und Kommunikationsformen zu, in denen das Wort, in welcher Bedeutung auch immer, eine nicht unbeträchtliche R olle spielt. D abei sollten wir allerdings das bisher $\mathrm{G}$ esagte im
A uge behalten, um eine Vorstellung zu haben von dem Wesen und dem Wert eines Wortes, wenn es glaubhaft vertreten, wenn ihm vorbehaltlos vertraut wird.

Es kann kaum daran gezweifelt werden, dass die Wahrnehmung des heutigen $M$ enschen wesentlich auf die Dinge des A Iltäglichen gerichtet ist, dass sich alle A ufmerksamkeit auf die Unmittelbarkeit des $\mathrm{G}$ egenwärtigen konzentriert. D ie A ufmerksamkeit gegenüber einer Sache, die für wichtig gehalten wird, führt zwangsläufig zur A usblendung anderer D inge, denen entsprechend ein geringerer Wert beigemessen wird bzw. die sich schließlich ganz unserer Wahrnehmung entziehen.

Wir verhalten uns dabei wie Schiffseigner, die den ganzen Tag damit verbringen und alle $M$ ühe daran setzen, ihr Schiff putzend, bemalend, lackierend, ausstaffierend und verzierend in einen vorzeigbar-makellosen Zustand zu versetzen. Vor lauter Glanz und Blendwerk ist es als Schiff kaum mehr zu erkennen. Das Studium der Nautik bzw. der Navigation ist völlig ins $\mathrm{H}$ intertreffen geraten, vom Willen, M ut und Wagnis, sich der H erausforderung des M eeres zu stellen, ganz zu schweigen, aus A ngst die Schönheit des Schiffes könnte Schaden leiden, der Lack Risse bekommen, die Planken angegriffen werden. Es ist ein Phänomen, dass der M ensch, der sein ganzes Augenmerk dem G lanz des A ugenblicks widmet und sich damit geradezu im Flüchtigsten überhaupt aufhält, dass dieser Mensch keine Aufwendungen und Mühen scheut, diesem Flüchtigen den Schein von D auer zu geben, indem er alles daran setzt, sein äußeres E rscheinungsbild makellos zu erhalten.

D as L eben gerät damit in die $\mathrm{E}$ indimensionalität des bloßen A nspruchs, der kurzlebigen B efriedigung, der opportunistischen Vorteilsnahme und des Bedürfnisses nach ängstlicher A bsicherung. $M$ an ist bemüht, den Status quo zu erhalten, ihn vor jeder schicksalhaften Beeinflussung zu bewahren, und man ist gewillt, sich dem L eben als Prozess, als A nspruch, als Sinn vermittelnde A useinandersetzung zu entziehen.

In dieser sehr eingeschränkten und vordergründigen Werteordnung haben wir das B ewusstsein und die A ufmerksamkeit für den verantwortlichen U mgang mit der Zukunft, mit der U mwelt, mit dem M itmenschen nahezu völlig verloren. D er G laube hat sich reduziert auf einen reinen Fortschritts- und Wachstumsglauben, auf einen Glauben, der sich ausschließlich auf das «M ehr» und damit auf die Steigerung und Intensivierung der A ugenblicksbefindlichkeit bezieht.

A lle Fragen, die sich mit der B edeutung und dem Sinn des Lebens befassen, die sich mit seiner Herkunft und seinem Ziel beschäftigen, die das $D$ enken über die eigene Begrenztheit hinaus erweitern, sind gleichermaßen verstummt. In diesem $\mathrm{R}$ ahmen, der von den Ü berlegungen über die B edeutung des Wortes definiert ist, erscheint es nicht angebracht, diese G edanken weiter zu verfolgen. Z wei sich daraus ergebende Konsequenzen können sich allerdings bei dem gestellten Thema als hilfreich erweisen.

Zum einen scheint die Vernachlässigung religiöser oder auch philosophischer O rientierungsbemühungen als Folge der überhand nehmenden materialistischen Nichtigkeiten und Augenblicksverliebtheiten Tendenzen zu fördern, welche die Beiläufigkeiten und 
A lltagsbesorgtheiten selbst in den R ang pseudoreligiöser B edeutsamkeit zu versetzen. D azu gehört auch, dass mit $E$ rsatzgottheiten und abergläubischen Selbstbespiegelungen versucht wird, entstandene Leerräume zu füllen. Es ist nicht zu übersehen, dass sich diese L eerräume bzw. die R äume, die auf ein existenzielles Vakuum hinweisen (V.E . Frankl), immer weiter ausbreiten.

Die zweite Konsequenz, die sich aus der beschriebenen Ich-verliebten D iesseitigkeit ergibt, zeigt sich darin, dass der M ensch, der die E rfüllung seines Lebens in der schnellen Sättigung durch das A ngebot des Augenblicks sucht, der die materiellen $G$ üter ausschließlich zur B efriedigung der eigenen B edürfnisse nutzt und der mit allen A nstrengungen und pseudoreligiösen Selbsttäuschungen versucht, seiner $K$ örperlichkeit Z eitlosigkeit und dem A ugenblick $B$ efriedigung zu verschaffen, dass dieser $M$ ensch früher oder später feststellen muss, dass ein so gelebtes Leben nicht wirklich befriedigen kann. Er wird erfahren, dass sich im allein materiell ausgerichteten Lebensverständnis zwangsläufig die L ebensinhalte verflüchtigen, dass in der zunehmend bewusst werdenden Vergänglichkeit alle Positionen des A ugenblicks ihre Bedeutung und ihren Wert verlieren.

E s stellt sich in diesem Z usammenhang die Frage, ob dem Wort angesichts der allgemeinen Wertematerialisierung überhaupt noch eine B edeutung zukommt.

E s kann kaum daran gezweifelt werden, dass die A nsprechbarkeit für das Wort weitgehend verloren gegangen ist; es ist geschehen, dass sich das Wort seiner geistigen Heimat entledigt hat; es ist in die Tiefen einer spröden Funktionalität abgeglitten und hat $E$ ingang gefunden in die sich hydraartig vermehrenden A mtstuben und in die Zirkuswelt von Selbstdarstellern und Claqueuren.

D ie ursprüngliche B edeutung des Wortes, die sich aus der A uthentizität, d.h. der unwiderlegbaren $E$ inheit von Wort und U rheberschaft herleitete, wurde dadurch relativiert, dass das Wort sich der Verbindlichkeit entledigte, sich aus dieser U rheberschaft auslöste und damit in eine B eziehungslosigkeit geriet, so wie ein Schiff, welches auf den $\mathrm{K}$ apitän verzichten muss.

A us der ursprünglichen $A$ uthentizität wurde $A$ ustauschbarkeit und B eliebigkeit; aus der A ussage wurde eine A bsicht; aus dem B und wurde (bestenfalls) ein Vertrag. Während sich ein Bund auf Vertrauen gründet, intendiert der Vertrag dazu, M isstrauen und $Z$ weifel zu zerstreuen. Während die A ussage einen Inhalt vermittelt, verfolgt die A bsicht ein Ziel. Während dem authentischen Wort schaffende K raft eigen ist, werden die Worte heute zum Strandgut der Zeit.

A uthentizität bedeutet, dass hinter einem gegebenen Wort die sprechende Person steht, ganzheitlich, untrennbar und unwiderruflich. Nur diese E inheit von Aussage und Sein rechtfertigt das vorbehaltlose Vertrauen, das dem $\mathrm{H}$ örer des Wortes die Möglichkeit gibt, sich auf dieses Wort zu verlassen.

Das Wort wird zum Mittler zwischen dem Wort-Geber und dem Wort-H örer. D ie E ntpersonifizierung des Wortes, die heute allenthalben zu bemerken ist, muss zu nachhaltigen Störungen im Bereich des $U$ mgangs und des Selbstverständnisses einer $G$ esellschaft führen, wobei sich alle Fehlentwicklungen auf die verloren gegangene M ittlerschaft des Wortes zurückführen lassen.
D as entpersonifizierte Wort, das Wort also, dem die Bürgschaft entzogen ist, ist leicht gesagt (um manches leichter als getan) und braucht nur selten den Beweis seiner Richtigkeit anzutreten. Die Wirkung, die es beabsichtigt, ist ihm oft wichtiger als die R ichtigkeit seines I nhalts.

Möglicherweise denken wir in diesem Zusammenhang an so oft gehörte politische R eden und die darin enthaltenen A ussagen und Versprechen. 0 der wir denken an das der Politik nahe stehende Stammtischszenarium, in dem sich Worte eifernd und sich gegenseitig übertrumpfend $\mathrm{G}$ ehör verschaffen. B ei Kongressen der verschiedensten A rt mag dies nicht anders sein. Bei genauerer Betrachtung allerdings bedienen sich unsere gesellschaftlichen $\mathrm{U}$ mgangsgepflogenheiten in zunehmendem $\mathrm{M}$ aße des leichten, auf Wirkung abzielenden Wortes. Selbst in akademischen K reisen wird der Versuchung, das Wort zu biegen und die Worte nach Belieben und nach beabsichtigter Wirkung einzusetzen, nicht immer widerstanden.

A lles aber, was nach Belieben genutzt wird, verliert zwangsläufig an Wert. Eine Spirale setzt sich in G ang, in der Weise, dass die B eliebigkeit des Wortgebrauchs die E ntwertung betreibt, so, wie die Entwertung des Wortes die B eliebigkeit seines $\mathrm{G}$ ebrauchs fördert. $\mathrm{D}$ ie heutigen Kommunikationsmaschinen, die bis in die intimsten Winkel unseres Lebens hinein ihre Dienste anbieten, verstärken diese Tendenz, indem sie das Wort der Seele berauben und seine Bedeutung auf die skeletthafte Funktion der Informationsvermittlung reduzieren. Es muss darum gehen, die E ntwertung der Worte, die wir stillschweigend und billigend hinnehmen, die lediglich von Zeit zu Zeit zum Gegenstand schöngeistiger Traktate gemacht wird, auf ihren Einfluss, auf die konkrete Situation des A Iltags hin zu überprüfen.

U m eine A bmachung gültig und beweiskräftig werden zu lassen, bedarf es des schriftlich formulierten Vertrags, dessen Richtigkeit und G ültigkeit durch die U nterschrift der Vertragspartner bestätigt wird. Wir haben uns daran gewöhnt, dass der $\mathrm{H}$ andschlag, al so das gegebene Wort, nicht mehr ausreicht, Verlässlichkeit zu gewährleisten. D ie Frage: «H aben Sie es schriftlich?» kennzeichnet ein Sicherheitsbedürfnis, welches alle Möglichkeiten der Täuschung, des Z weifels und missliebiger Interpretationen von vornherein auszuschließen versucht.

Auch das Vertragswerk selbst spiegelt dieses allgemeine B edürfnis nach $A$ bsicherung, indem jede $M$ öglichkeit einer einseitigen Vorteilsnahme ausgeschlossen und jeder nur erdenkliche M issbrauch durch vertragliche Festschreibung verunmöglicht werden soll. So sind wir gewohnt, dass Verträge neben den aussagekräftigen A bhandlungen über den Vertragsinhalt eine Menge von klein gedruckten Zusätzen und Ergänzungen beinhaltet. Auf dieses «K leingedruckte» werden wir meist erst dann aufmerksam, wenn eine Situation eingetreten ist, die die Gültigkeit des Vertrags in Frage stellt. Wohl keiner denkt daran, am wenigsten zum Zeitpunkt der Vertragsunterzeichnung, dass ein solcher Fall je eintreten könnte.

Was wir im Zusammenhang mit dem Vertragswesen gewohnt sind hinzunehmen, hat längst schon Eingang gefunden in unseren alltäglichen U mgang mit dem Wort. Wir sagen das «G roßgedruckte» 
und legen uns im Innersten schon manch «K leingedrucktes» für eine eventuelle spätere R echtfertigung zurecht. Je umfangreicher das «K leingedruckte» zur A bsicherung gedacht wird, desto weniger eindeutig, klar und verlässlich braucht die Aussage des «G roßgedruckten», also des G esagten, zu sein.

So werden Aussagen getroffen und Behauptungen aufgestellt, während ihre Z weideutigkeit oder gar U nrichtigkeit durch das nicht Gesagte von vornherein billigend in Kauf genommen wird. D em Wort wird damit der Boden für das Vertrauen, die Nachhaltigkeit und den Wahrheitsbezug entzogen, es ist freigegeben für jedwede K alkulation hinsichtlich Wirkung, Veruntreuung und Täuschung. Die Verführung dazu beginnt im Kleinen, mit dem zurechtrückenden «corriger la fortune» und reicht bis hin zur bewussten Falschaussage, immer hoffend, dass die H intertür des «K leingedruckten» nicht benutzt werden muss und man nicht gezwungen ist, die verdeckten M anipulationen offen zu legen.

$D$ ie $D$ estabilisierung des Wortes ist allenthalben spürbar, nicht nur in der Politik und in der Wirtschaft, sondern auch in der Wissenschaft, in den M edien und selbstverständlich im täglichen U mgang mit dem Wort. Dabei geht es um Versprechen, die wohlweislich nicht gehalten werden können. E s geht um Fälschung von E rgebnissen mit klar erkennbarer Wirkungsabsicht, es geht um unkritische Selbstpositionierungen mit dem Ziel der eitlen Selbstdarstellung, der ausweitenden E influssnahme und des kommerziellen $\mathrm{Ge}$ winns, es geht um die D enaturierung des Wortes selbst, welches als Schlagwort und als Parole genutzt wird, um mit dem inm eigenen Verführungspotential vorteilhafte Stimmungen gegen das $R$ ational und gegen differenzierte A rgumentationen zu erzeugen.

Diese B eispiele haben eines gemeinsam, sie versuchen mit dem Wort eine Wirkung zu erzielen, nicht aber einen Wahrheitsgehalt authentisch zu vertreten bzw. verantwortlich zu vermitteln.

$D$ as Wort hört auf Vermittler von Leben spendender Verlässlichkeit zu sein, es trägt vielmehr in diesem verwaisten $D$ asein zur Verunsicherung, zur A rrosion und schließlich zur Auflösung des Lebens bei.

O bwohl zu wenig bewusst, liegen die sich daraus ergebenden Folgen auf der $\mathrm{H}$ and. Es sind dies 1 ) die Entwertung und der M issbrauch des Wortes, 2) das Ü berhandnehmen anonymisierender Sicherheits- und Kontrollmechanismen und schließlich 3) die zunehmende Vereinsamung des $M$ enschen.

Ad 1) Der Verlust an Glaubwürdigkeit und Verbindlichkeit des Wortes fördert seinen beliebigen und ungedeckten Gebrauch. Z wangsläufig erfährt es mit dieser E ntwertung eine inflationäre Vervielfältigung und eine inhaltlose Vermassung. M it immer mehr Worten wird immer weniger gesagt, immer mehr Zeit wird mit Nichtigkeiten aller A rt verbracht; mit einer M enge unbedeutender Worte wird der fehlende Inhalt kaschiert.

D ie entstehende E intönigkeit ergötzt sich am Trivialen, die L angeweile sucht das Banale, um das Innere zu betäuben. M it der $E$ ntwertung des Wortes geht gleichzeitig die Tendenz seines bewussten oder unbewussten $M$ issbrauchs einher. $M$ it dem leichten und oft entstellten Wort wird geworben, gelockt und verführt. Das Wort wird zur alleinigen Wirkung missbraucht. Worte bilden den Rahmen für den immer mehr verloren gehenden Inhalt. Weil der In- halt immer bedeutungsloser wird, kann er verändert, manipuliert und verfälscht werden, scheint es doch gesichert, dass es mit plakativen und blumenreichen Wortattraktionen gelingt, jeden wie auch immer geschändeten Inhalt an den M ann zu bringen.

Nun sind ja Worte nichts anderes als Spiegel unserer selbst, als Spuren, die wir durchs L eben gehend hinterlassen. So spiegeln die Worte unserer D enk- und Lebensart, und die Spuren, die wir hinterlassen, deuten mit ihrer U nstetigkeit auf eine um sich greifende Plan- und O rientierungslosigkeit. Nicht weniger aber auf U nsicherheit und $\mathrm{H}$ altlosigkeit, die mit der Beliebigkeit des Wortes einhergeht.

Da ist ein Journalist, der die Gunst der Stunde nützt und einen recht üblichen Vorgang schreibend zur A ttraktion werden lässt; da ist ein «Prominenter» der hofft, seinem L eben dadurch eine Bedeutung verschaffen zu können, dass er es auf unzählige gehaltlose Buchseiten ausbreitet; da ist ein Mediziner, der meint, in der Heilkunst schreibend und publizierend unter Verzicht auf Praxis- und Patientennähe reüssieren zu können; da ist ein Politiker, der versucht, mit wortreichen Versprechungen von der Wirklichkeit abzulenken und sich die Gunst seiner Wähler zu verschaffen; da ist ein Priester, der das Wort zur tradierten Formel und zur seelenlosen R eliquie verfremdet, statt es in der Wahrheit lebendig werden zu lassen.

Ad 2) Der Verlust an Glaubwürdigkeit und Verbindlichkeit des Wortes fördert den Verlust an Verlässlichkeit und Sicherheit im persönlichen und gesellschaftlichen U mgang. Das menschliche Streben ist aber wesentlich auf Sicherheit und Vermeidung von R isiken gerichtet. Wenn dem $M$ enschen das Wort nicht mehr verlässlich erscheint und inm das Vertrauen auf das Wort abhanden gekommen ist, beginnt er, Kontrollmechanismen zum A usschluss von $\mathrm{M}$ issbrauch und etwaigen Risiken zu ersinnen. Das heutige $\mathrm{Ge}$ sundheitswesen ist ein beredtes B eispiel für die konsequente und spiralartige Entwicklung vom authentischen Wort hin zur anonymisierenden Bürokratie, für eine Entwicklung vom Wort, welches dem Wesen nach immer persönlich ist, hin zur seelenlosen Medizinverwaltung.

$M$ it großem bürokratischem A ufwand und mit einem Ü berangebot technischer Perfektion wird versucht, den zunehmenden Vertrauensverlust aufzufangen. D ass sich dabei aber eine Spirale auftut, in der sich das Vertrauen immer mehr verliert und schließlich eine Situation eintritt, in der jede persönliche Bindung durch formale Elemente einer anonymen B ürokratie und einer Pseudo-Sicherheit vermittelnden Technik ersetzt wird, ist kaum bewusst.

D ie angestrebte Sicherheit wird zum einklagbaren R echt, wodurch endgültig das, was anfangs den Bund des Lebens ausmachte, von der Eigenwilligkeit des egozentrischen A nspruchs getilgt wird. Die Entidealisierung der Medizin löst den M enschen aus jeder Form der Schicksalhaftigkeit heraus, aus jedem übergeordneten Seinsund Sinnverständnis und macht inn damit zur Produkt der reinen B erechenbarkeit. D ie M edizin wird zum Verwaltungssyndikat verfremdet, sie gerät unreflektiert in die M achbarkeitsideologie der Technik und wird durch ihre U nterwerfung unter das D iktat ökonomischer $G$ esetze zum G eschäft.

Was für die M edizin gilt, hat nicht weniger $\mathrm{G}$ ültigkeit für alle Bereiche unserer gesellschaftlichen Ordnung. Es ist der M ensch, der 
sich aus dem B und der Schöpfung ausgegrenzt hat und dabei Gefahr läuft, seine eigene I dentität zu verlieren. E s ist der M ensch, der die Bürgschaft für das Wort aufgegeben hat und damit zunehmend der Sprachlosigkeit verfällt. So, wie oft Belanglosigkeiten und $\mathrm{N}$ ichtigkeiten des A lltags in den Rang pseudoreligiöser Bedeutsamkeit erhoben werden, so gerät der regulierende Charakter der Verwaltung in eine unausweichliche Dominanz, die Idee zerschlagend, die A bsolutheit beanspruchend. Ü berall dort, wo der $M$ ensch als $M$ ensch versagt, regieren anonyme und gefühllos regulierende $\mathrm{K}$ räfte. $\mathrm{M}$ it dem Wort stirbt die I dee. M it jeder sterbenden Idee verlieren wir ein Stück Kultur.

Ad 3) Der Verlust an Glaubwürdigkeit und Verlässlichkeit des Wortes führt zu einer $E$ ntzweiung des $M$ enschen, zu einer E ntkoppelung und $A$ uflösung seiner ureigensten Bindungsfähigkeit.

$M$ an stelle sich ein G las Wasser vor, in dem eine Substanz gelöst ist. Durch die elektrische Ladung der Moleküle werden diese in $\mathrm{L}$ ösung gehalten. $\mathrm{M}$ it ihren $\mathrm{K}$ raftfeldern treten sie in gegenseitige Interaktion, sie ziehen sich an und stoßen sich ab; alles ist in einer gehaltenen B ewegung. Was für die M oleküle das elektrische Feld ist, das ist für den M enschen in seiner zwischenmenschlichen Interaktion das Wort. Es bindet, und es wird gelöst, es stabilisiert die interaktiven gesellschaftlichen L ebensprozesse.

E ntzöge man den M olekülen ihr elektrisches Feld, dann fielen die M oleküle sedimentierend zu B oden, willenlos der Schwerkraft folgend. Nichts anderes würde geschehen - oder geschieht - , entzöge man die stabilisierende $\mathrm{K}$ raft des Wortes. $\mathrm{D}$ ie M enschen verhielten sich wie eine willenlose Masse, fremdbestimmt durch die Kräfte der Zeit, der M ode, des A ugenblicks.

Vor dem Hintergrund seiner ureigensten B edeutung vermittelt das Wort mit seiner Verbindlichkeit, seiner K larheit und E indeutigkeit in der Z one des persönlichen A ngesprochen-Seins gesicherte Positionen hinsichtlich O rientierung, Z ugehörigkeit und wertigen SeinVerstehens. Die im Wort enthaltene Zusage ist demzufolge die wichtigste Voraussetzung für die Freiheit eines Menschen, für seine U nabhängigkeit, seine $E$ igenverantwortlichkeit und $E$ igenständigkeit.

Nur aus der Freiheit und der E igenständigkeit eines M enschen heraus können Verbindlichkeiten entstehen und Valenzen, die über das Vergängliche und die L aunen des A ugenblicks hinaus B estand haben. Wenn nun das Wort an Glaubwürdigkeit verliert, wenn es in die Trivialität abgleitet und nur noch auf Wirkung und Clownerie bedacht ist, dann gerät der Mensch in eine verhängnisvolle O rientierungslosigkeit, die ihn abhängig werden lässt von vordergründigem Streben nach Sicherheit, von E igenliebe und allen Formen der Fremdbestimmung.

Wer dem Wort nicht vertraut, der wird auch kein verlässliches Wort geben können. Wer ohne I dee lebt, lebt nur noch sich selbst, lebt nur noch dem A ugenblick ohne eine über sich selbst hinausreichende Perspektive; er rudert in den Nichtigkeiten des A lltäglichen ohne Ziel außer dem, sich selbst zu genügen. Jede menschliche Beziehung ebenso wie jede Ausrichtung auf eine Sache, auf eine Vorstellung oder auf eine Zielsetzung muss unernst und unverbindlich bleiben, so lange sie nicht von der Verlässlichkeit der Zusage bzw. der Verbindlichkeit des Wortes getragen ist.

D ie Konsequenz aus all dem ist nicht zu übersehen: es ist ein Stück Vitalität und L ebenszuversicht verloren gegangen.

E s gilt, die B edeutung des Wortes wieder zu erkennen; E s gilt, die Kultur seines verlässlichen U mgangs neu zu entdecken. E s gilt, einen Weg zu finden aus der von Sprachlosigkeit gekennzeichneten Isolation. 